\title{
APORTACIONES DE LA PSICOTERAPIA INTEGRATIVA AL ESTUDIO Y COMPRENSIÓN DEL DESARROLLO HUMANO
}

\section{CONTRIBUTIONS OF INTEGRATIVE PSYCHOTHERAPY TO THE STUDY AND UNDERSTANDING OF HUMAN DEVELOPMENT}

\author{
Esther Zarandona de Juan
}

Departamento de Psicología Evolutiva y de la Educación, Universidad del País Vasco, España ORCID: https://orcid.org/0000-0002-5756-7599

Cómo referenciar este artículo/How to reference this article:

Zarandona, E. (2021). Aportaciones de la Psicoterapia Integrativa al estudio y comprensión del desarrollo humano. Revista de Psicoterapia, 32(118), 7-24. https://doi.org/10.33898/rdp.v32i118.482

\section{Resumen}

En este artículo se presentarán los resultados preliminares de un trabajo sobre las aportaciones de la Psicoterapia Integrativa al estudio y comprensión del desarrollo humano tomando como referencia la perspectiva constructivista sociocultural del desarrollo, -también denominada histórico-cultural o cultural-. Se basa en el estudio de seminarios experienciales y didácticos de formación en esta línea de psicoterapia. Las técnicas de recogida de datos han sido la observación participante, el registro de sesiones individuales de psicoterapia con los participantes y el análisis de documentos. Entre las conclusiones que se van perfilando cabe destacar el conocimiento que los métodos de la Psicoterapia Integrativa proporcionan sobre la acción del propio sujeto y su vivencia en el proceso de internalización o apropiación de lo que aparece en el plano interpsicológico al intrapsicológico.

Palabras clave: desarrollo, psicoterapia integrativa, perspectiva sociocultural, interacción, vivencia

\begin{abstract}
This article will show the preliminary results of the Integrative Psychotherapy contributions to the study and understanding of human development taking as reference the constructivist sociocultural perspective of development, -also known as historical-cultural or cultural-. It is based on the study of experienced and didactical seminars of training in this psychotherapy line. The data collection techniques have been the participant observation, individual session registry of psychotherapy with the participants and document analysis. Among the conclusions that are outlining it should be noted the knowledge that Integrative Psychotherapy methods provide about the action of the subject himself and his experience in the appropriation process of what appears in the interpsychological to the intrapsychological plane.
\end{abstract}

Keywords: development, integrative psychotherapy, sociocultural perspective, interaction, experience

Fecha de recepción: 12 de octubre de 2020. Fecha de aceptación: 24 de noviembre de 2020.

Correspondencia sobre este artículo:

E-mail: esther.zarandona@gmail.com

Dirección postal: Calle Fika 11, 4 izq. 48006 Bilbao, Bizkaia, España

(C) 2021 Revista de Psicoterapia 
El origen de este trabajo está en las observaciones que recogí en los primeros seminarios experienciales de formación en Psicoterapia Integrativa. Me llamó poderosamente la atención el rigor en el estudio y tratamiento de la interacción terapéutica que pude observar porque estaba muy en la línea de los trabajos de investigación sobre la interacción social en contextos familiares y educativos de los que partía mi propia investigación en ese campo (Zarandona, 2007, 2011, 2015), trabajos todos ellos realizados desde la perspectiva constructivista sociocultural del desarrollo y la educación.

Una perspectiva muy “viva”, por decirlo así, en lo que estaba observando en la teoría y la práctica de la Psicoterapia Integrativa: gran parte de la creación teórica de la perspectiva constructivista sociocultural del desarrollo, así como muchos de los resultados de las investigaciones realizadas desde ese marco se desplegaban ante mi vista en la acción, o mejor, en la inter-acción, de los protagonistas de las sesiones individuales dentro del grupo que se desarrollaba durante los seminarios.

Empecé entonces a vislumbrar el interés de considerar la práctica clínica llevada a cabo desde la Psicoterapia Integrativa como otro contexto de desarrollo y el interés en consecuencia de estudiar la interacción social de este contexto, así como las aportaciones de esta forma de trabajo terapéutico para la comprensión de los procesos de desarrollo a lo largo del ciclo vital.

Así pues, con el objetivo de estudiar la práctica clínica desde esta perspectiva evolutiva preguntándome sobre sus supuestas aportaciones al estudio y comprensión del desarrollo humano a lo largo del ciclo vital, comencé a tomar notas sobre el desarrollo de cada seminario y registros literales, -algunos en audio y otros escritos-, de las sesiones de psicoterapia individuales que tenían lugar en los mismos y también a estudiar y analizar desde esta perspectiva diferentes documentos (libros, artículos) publicados sobre los métodos de la Psicoterapia Integrativa. Lo que se presenta a continuación es el resultado de una primera aproximación analítica, -provisional, por tanto-, a algunos de los datos recogidos, en concreto las categorías de interpretación que se van perfilando. Previamente, se mostrarán los conceptos nucleares de la perspectiva constructivista sociocultural del desarrollo para poder entender la mirada que ha guiado el trabajo.

\section{La Perspectiva Constructivista Sociocultural}

\section{Construimos y lo Hacemos en Relación}

En este enfoque teórico se parte de la idea compartida por todas las posiciones constructivistas de los seres humanos como constructores activos (Cubero, 2005). La perspectiva sociocultural, -también denominada cultural o histórico-cultural-, cuyo fundamento y fuente principal de inspiración es el pensamiento de Vygotski, tiene en cuenta que los procesos de construcción no tienen lugar en soledad, se dan siempre en relación y son deudores de la sociedad y cultura a la que pertenecen las personas. 
Parte de la consideración de que la evolución que experimentamos los seres humanos desde el nacimiento hasta la muerte es, sobre todo, un producto de las interacciones que se establecen al participar en la actividad sociocultural entre el sujeto que aprende y los agentes mediadores de la cultura, entre los cuales los educadores (familias, profesores, etc.,) ocupan un lugar esencial (Coll y Solé, 1990). La interacción social en esta visión teórica no se reduce, por tanto, a una variable más a incorporar a determinados modelos de funcionamiento individual.

\section{El Origen de las Funciones Psicológicas Superiores}

La visión del psicólogo ruso queda claramente reflejada en su conocida ley de la doble formación de los procesos psicológicos superiores o ley genética del desarrollo cultural (Vygotski, 1978/1979):

En el desarrollo cultural del niño, toda función aparece dos veces: primero, a nivel social, y más tarde, a nivel individual: primero entre personas (interpsicológica), y después en el interior del propio niño (intrapsicológica). [...]. Todas las funciones superiores se originan como relaciones entre seres humanos. (p. 94)

Ya desde hace tiempo, investigaciones posteriores a Vygotski han mostrado que "el bebé humano nace social en el sentido de que su desarrollo dependerá desde el principio de los patrones de interacción con los mayores” (Kaye, 1982/1986, p. 41-42). Desde esta concepción entonces:

[...] el desarrollo humano es una construcción conjunta en la que el sujeto es únicamente un vértice del triángulo sujeto-objeto-“otro social”, de modo que el desarrollo psicológico se construye a través de las interacciones sujeto-objeto cuando “alguien” organiza socialmente la actividad del sujeto con el objeto de conocimiento. (Vila, 1996, p. 89)

En este punto, es necesario precisar que pese a lo que el término de "sociocultural” y lo que se viene explicando hasta ahora puedan sugerir, -una primacía de los "elementos sociales" por ejemplo-, este enfoque, tal como nos recuerda Barbara Rogoff (1990/1993):

Más que otorgar primacía al papel de la actividad social o al individuo, el objetivo es reconocer que el papel desempeñado por la herencia de un grupo social, la participación en él y los esfuerzos individuales son fundamentales e inseparables. (p. 51)

\section{El Vínculo Irreductible entre Mente y Cultura}

Desde la visión que acabamos de esbozar, resulta entonces incompleto centrarse sólo en la relación entre el desarrollo individual y la interacción social sin tener en cuenta la actividad cultural en la que tienen lugar las acciones individuales e interpersonales (Rogoff, 1997). La perspectiva sociocultural sostiene que:

La construcción de la mente individual y su integración en un contexto social y cultural son procesos interdependientes que forman una unidad 
indisoluble, de modo que la construcción del yo es inseparable del contexto social y cultural en que se produce. (Vila, 1995, p. 3)

Para Vygotski es fundamental el papel mediador de la cultura en la formación de la mente ( Martí, 2005; Santamaría, 2004; Vygotski, 1978/1979; Wertsch, 1985/1988). En esta perspectiva, se entiende que la mente crea y, a la vez, tiene su origen en la cultura humana. En esta posición, el punto central es la construcción de significado. Así, el significado es entendido como el vínculo entre mente y cultura: gracias a la construcción del significado podemos situar de forma adecuada las cosas, estados, acciones, etc., en los contextos culturales de la realidad para comprender qué son. De manera que los significados están "en la mente", pero su origen se sitúa en la cultura, por ello se construyen a través de su negociación y, en último término, de la comunicación (Bruner, 1983/1986, 1990/1991, 1997, 2008; Vygotsky, 1982/1993). El conocimiento y la comunicación son en consecuencia dos procesos inseparables con un funcionamiento interdependiente.

\section{La Consideración del Contexto, ese Tejido}

Este cambio de perspectiva radical supone asumir hasta las últimas consecuencias que "para calificar un estudio como sociocultural, $[. .$.$] lo que resulta esencial$ es que la situcionalidad del funcionamiento mental sea reconocida y analizada de alguna forma" (Wertsch, 1997, p. 49) porque el objetivo de la investigación realizada desde esta perspectiva es "comprender la relaciones entre el funcionamiento mental humano, por un lado, y la situación cultural, histórica e institucional, por el otro" (p. 49). La asunción es, por tanto, que construimos el conocimiento en relación y de una manera situada, esto es, contextualizada. Hablar de situcionalidad nos lleva a adentrarnos en el concepto de contexto. Es ésta una noción resbaladiza, nada fácil de "atrapar" conceptual y metodológicamente hablando, aunque intentos no faltan y dificultades tampoco. Sin entrar en ellos, es imprescindible por lo menos mencionar el modelo ecológico del desarrollo humano que describe los diferentes sistemas en los que se inserta el desarrollo subrayando que lo importante es la experiencia del sujeto en dichos sistemas (Brofenbrenner, 1979/1985, 1979/1987), muy en consonancia como veremos con la perspectiva sociocultural; los estudios basados en la consideración del contexto como contexto social e institucional de actividad (Wertsch, 1985/1988), actividad sociocultural (Rogoff, 1997, 2012), escenario sociocultural o comunidad de práctica (Cubero 2005); el contexto como construcción conjunta de los participantes en una actividad; el contexto como tejido (Cole, 1994) del que formamos parte y el contexto como fenómeno mental en el sentido de significados compartidos (Mercer, 2000/2001).

\section{En Relación, pero ¿Cómo?}

Hasta ahora hemos dicho que, participando en las diferentes actividades socioculturales, en interacción con el resto de participantes en ella. Pero falta aclarar cómo se realiza la transición del funcionamiento interpsicológico al intrapsicológico. 
En lo que sigue se expondrán algunas de las herramientas conceptuales creadas desde la perspectiva sociocultural para responder a estas preguntas.

\section{Internalización y Apropiación}

Una de esas herramientas es el concepto de internalización referido al proceso mediante el cual se produce la transformación de las actividades socioculturales o fenómenos sociales en fenómenos psicológicos. Vygotski llama internalización a "la reconstrucción interna de una operación externa" (1978/1979, p. 92). Para él "la internalización de las actividades socialmente arraigadas e históricamente desarrolladas es el rasgo distintivo de la psicología humana” (p. 94). Desde su punto de vista, son las propiedades de esos procesos socioculturales las que nos permiten entender la aparición del funcionamiento interno:

En situaciones concretas, Vygotski defiende la existencia de un isomorfismo entre la organización de los procesos en los dos planos y, en cualquier caso, afirma que la estructura del funcionamiento interpsicológico tiene un enorme impacto sobre la estructura del funcionamiento intrapsicológico resultante. (Wetsch, 1985/1988, p. 78)

Nos equivocaríamos, no obstante, si viéramos en este planteamiento un desprecio por la importancia de la agencia de las personas. Al contrario: esta interiorización ha de ser entendida no como una copia de los procesos inter-psicológicos externos, sino como una verdadera construcción del plano intra-psicológico (Wertsch, 1998/1999) que implica cambios en las estructuras que se interiorizan. Según esta visión, aunque el origen de las funciones superiores esté en las relaciones que se establecen entre las personas, la actividad de las personas es sumamente importante para que tenga lugar el desarrollo: son éstas las que realizan la transformación en sus características individuales, "las cuales formarán parte de su particular bagaje psicológico por medio de la interiorización o internalización de lo que, en un primer momento, ha aparecido en el seno de sus contactos y vivencias con las personas que interactúan con ellas” (Vila, 1995, p. 30). Vygotski (1982/1993) y Leontiev (1983) utilizaron también el término apropiación para referirse a esta reconstrucción.

\section{Zona de Desarrollo Próximo, Andamiaje y Participación Guiada}

El concepto de Vygotski de zona de desarrollo próximo es el complemento de su ley de la doble formación de los procesos psicológicos superiores en el que ilustra el papel de la interacción en el desarrollo y trata de dar respuesta a cómo se da la transición de lo interpersonal a lo intrapersonal. Lo define de esta manera: [...] No es otra cosa que la distancia entre el nivel real de desarrollo, determinado por la capacidad de resolver independientemente un problema, y el nivel de desarrollo potencial, determinado a través de la resolución de un problema bajo la guía de un adulto o en colaboración con otro compañero más capaz.

[...] Si un niño es capaz de realizar esto o aquello de modo independiente, 
significa que las funciones para tales cosas han madurado en él. Entonces, ¿qué es lo que define la zona de desarrollo próximo, determinada por los problemas que los niños no pueden resolver por sí solos, sino únicamente con la ayuda de alguien? Dicha zona define aquellas funciones que todavía no han madurado, pero que se hallan en proceso de maduración, funciones que en un mañana próximo alcanzarán su madurez y que ahora se encuentran en estado embrionario. Estas funciones podrían denominarse "capullos" o "flores" del desarrollo, en lugar de "frutos" del desarrollo. El nivel de desarrollo real caracteriza el desarrollo mental retrospectivamente, mientras que la zona de desarrollo próximo caracteriza el desarrollo mental prospectivamente. (Vygotsky, 1978/1979, pp. 133-134)

Para Vygotski, por tanto, la educación es una de las fuentes más importantes del desarrollo: es la mediadora entre la cultura y el desarrollo.

La metáfora del andamiaje se sitúa entre las investigaciones que arrojan alguna luz sobre cómo niños y niñas progresan a través de las zonas de desarrollo próximo que se crean en las interacciones educativas con otras personas más capaces. Al igual que cuando se construye un edificio se colocan una serie de apoyos y soportes que luego, progresivamente, se van retirando, el concepto de andamiaje se refiere al hecho de que, al principio de la resolución de una tarea conjunta, el adulto aporta una serie de ayudas que, poco a poco, va eliminando a medida que el sujeto que aprende se vuelve más independiente en la resolución individual de la tarea. Este concepto fue empleado inicialmente por Wood et al. (1976) con el objeto de caracterizar el tipo de ayudas que recibe el aprendiz de los adultos y el ajuste de las mismas. La metáfora del andamiaje pone de relieve la transferencia de control y responsabilidad de parte del adulto hacia el aprendiz cuando ambos están implicados conjuntamente en una actividad y sigue siendo fuente de inspiración en numerosas investigaciones que estudian este proceso de transferencia de control y responsabilidad (Zarandona, 2015).

Por último, según Barbara Rogoff (1990/1993, 2012), la participación guiada es un proceso en el que "los papeles que desempeñan el niño y el cuidador están entrelazados, de tal manera que las interacciones rutinarias entre ellos y la forma en que habitualmente se organiza la actividad proporcionan al niño oportunidades de aprendizaje tanto implícitas como explícitas" (Rogoff, 1990/1993, p. 97).

En su análisis, Rogoff (1990/1993) aporta datos interesantes sobre los papeles de los agentes en la participación guiada. Respecto a los niños y niñas constata que su papel es muy activo cuando se sirven de la guía social: son los pequeños los que proporcionan al adulto la guía necesaria para que éste pueda a su vez guiarles adecuadamente. En cuanto a la participación de los adultos, nuestra autora resalta los procesos que se orientan a tender puentes desde lo conocido a lo nuevo y a estructurar las actividades infantiles y transferir la responsabilidad. Aquí Rogoff, destaca el ajuste del apoyo del adulto. Éste ajusta el grado de apoyo durante la interacción en base a las "pistas" que aparecen en la misma de forma que le hace 
posible alcanzar "una estructura desafiante y, a la vez, de apoyo, que se ajuste a los cambios en la comprensión del aprendiz” (p. 142). Los niños por su parte tienen un papel activo dirigiendo el apoyo de los adultos y ajustándolo a medida que van desarrollando sus propias destrezas.

\section{Mediación Semiótica e Intersubjetividad}

La mediación es fundamental en la teoría histórico-cultural. En la concepción de Vygotski (1978/1979, 1982/1991a, 1982/1991b, 1983/1995) la actividad humana es un fenómeno mediado por signos y herramientas. En este contexto, emplea el término de función psicológica superior, o conducta superior, para referirse a la combinación de herramienta y signo en la actividad psicológica (1978/1979, 1983/1995). Como señala Wertsch, esta concepción es central en análisis de los procesos psicológicos realizado por Vygotski pues consideraba el desarrollo “en términos de transformaciones cualitativas fundamentales o 'revoluciones' en cambios en las herramientas psicológicas” (Wertsch, 1985/1988, p. 95).

A partir de esta concepción instrumental es desde donde se entiende su ley de la doble formación en la que afirma que toda función aparece primero a nivel social y su concepción de la internalización como reconstrucción interna de lo que ha aparecido previamente en el nivel social, como se ha explicado en los apartados precedentes.

Wertsch (1985/1988, 1989, 1991/1993) opina que la consideración de las herramientas psicológicas como sociales por lo que tienen que ver con el fenómeno social de la comunicación cara a cara y la interacción social, tiene importantes consecuencias para la teoría de Vygotski sobre los procesos psicológicos humanos dado que:

Por ejemplo, si la función principal de las herramientas psicológicas tales como el lenguaje es la de comunicar, es razonable suponer que estos instrumentos de mediación se formen de acuerdo con las demandas de la comunicación. Si estos instrumentos desempeñan, además, un papel importante en la configuración de los procesos psicológicos de los individuos, podemos esperar que estos procesos se configurarán indirectamente a partir de las fuerzas que se originan en la dinámica de la comunicación. (Wertsch, 1985/1988, p. 97)

Por esta razón se han realizado numerosos trabajos que estudian sobre todo los mecanismos semióticos implicados en el establecimiento, desarrollo y finalización de las actividades conjuntas adulto-niño y sobre estos mecanismos semióticos también a partir de la noción de intersubjetividad, término que se refiere a la mutua comprensión que logran las personas que se comunican, noción empleada precisamente por Rogoff (1990/1993) para analizar el proceso de tender puentes de lo conocido a lo nuevo al que nos hemos referido en el apartado anterior.

Estos trabajos se interesan por las propiedades de los procesos interpsicológicos que permiten la transición al plano intrapsicológico partiendo del presupuesto 
vygotskiano de que el funcionamiento interpsicológico está indisolublemente ligado al funcionamiento intrapsicológico. La cuestión que interesa resaltar es que en la transición del funcionamiento interpsicológico al intrapsicológico cualquier cambio en el primero ocasiona un cambio correspondiente en el segundo, pero no de una manera repentina y bien definida, pues entonces estaríamos olvidando su dinámica, esto es: "que en el plano interpsicológico se producen una serie de cambios y que cada uno de éstos queda reflejado en un cambio en el funcionamiento intrapsicológico" (Wertsch, 1985/1988, p. 169).

De los estudios que se han realizado analizando la interacción adulto-niño se confirma que un cambio en el plano interpsicológico necesariamente va asociado a un cambio en el plano intrapsicológico y llevan a una conclusión muy relevante desde el punto de vista de la investigación en este campo: “(...) al caracterizar el funcionamiento interpsicológico en una tarea, necesariamente se caracteriza el funcionamiento intrapsicológico. En realidad, este procedimiento proporciona información sobre los procesos individuales que a menudo no puede obtenerse por otras vías” (Wertsch 1985/1988, pp. 176-177).

Por otra parte, sobre la intersubjetividad estudiada en el proceso de tender puentes de lo conocido a lo nuevo en la participación guiada, Rogoff (1990/1993) afirma que "esa comprensión tiene lugar entre personas; no puede atribuirse a una u otra persona de las que participan en la comunicación” (1990/1993, p. 99). En cuanto a los orígenes de ésta, Rogoff pone de relieve que:

Los niños pequeños son tan hábiles a la hora de obtener información de las miradas, las muecas y el humor de los adultos que uno de los grandes retos, en el momento de hacer pruebas escolares, es evitar acciones que pueden ser interpretadas como pistas. Los niños las piden con insistencia, y utilizan tales claves, incluso cuando han de resolver test de inteligencia estandarizados. (1990/1993, p. 99)

Rogoff (1990/1993) por otra parte destaca el papel de la comunicación emocional y no verbal a la hora de establecer esa comprensión mutua en la que consiste la intersubjetividad.

\section{Finalmente, la Vivencia}

Vygotski desarrolló otro concepto, muy a tener en cuenta en el tema de la transición de lo interpsicológico a lo intrapsicológico. Nos referimos a la vivencia (Vygotski, 1984/1996). Según Vygotski, en concordancia con los planteamientos de la psicología ecológica, el papel del entorno en el desarrollo solamente se puede conocer cuando se conoce la relación del niño con él y esta relación -el significado que tiene el entorno para el niño-, está mediada por la experiencia emocional o vivencia del niño. Con el concepto de vivencia Vygotski hizo referencia a la integración entre los componentes afectivo e intelectuales de la conciencia (1982/1993, 1984/1996). Tal como señala una de sus alumnas, Lidia Ilínichna Bozhovich, Vygotski había comenzado a examinar la cuestión de la necesidad de integrar los 
fenómenos afectivos e intelectuales en el estudio del funcionamiento humano al final de su vida, pero su muerte cortó de raíz la investigación (Wertsch, 1985/1988; Fariñas, 1999b). Bozhovich y sus colegas tomaron esta tarea como una de sus principales inquietudes de investigación. Otros discípulos de Vygotski como Leontiev (1983), basándose en el desarrollo incipiente del tema por parte de su maestro y por posteriores desarrollos de sus ideas realizados por sus seguidores, se centraron en cómo la afectividad proporciona las fuerzas integradores y motivacionales de la conciencia (Wertsch, 1985/1988), aunque según Fariñas (1999a) -psicóloga cubana doctorada bajo la dirección de Galperin-, tampoco dieron continuidad a las sugerencias de Vygotski hasta sus últimas consecuencias (Fariñas, 1999a). Por el contrario, la cátedra de Vygotski de la Universidad de La Habana dirigida por Guillermo Arias y formada por psicólogos que estudiaron y realizaron sus tesis doctorales con discípulos directos de Vygotski (Arias, 2015), se distingue por la prominencia que otorga a esta categoría tanto en su explicación del enfoque histórico-cultural como en los trabajos desarrollados desde ahí (Fariñas, 1999a, 1999b, 2013, 2015; Marcos, 2005; Corral, 2006); y en los últimos años se le está empezando a prestar atención entre nosotros (Esteban, 2011; Zarandona, 2015).

Para Vygotski la unidad de análisis adecuada para el estudio de la personalidad y el medio es la vivencia:

La vivencia del niño es aquella simple unidad sobre la cual es difícil decir que representa la influencia del medio sobre el niño o una peculiaridad del propio niño. La vivencia constituye la unidad de la personalidad y del entorno tal como figura en el desarrollo. Por tanto, en el desarrollo, la unidad de elementos personales y ambiéntales se realiza en una serie de diversas vivencias del niño. La vivencia debe ser entendida como la relación interior del niño como ser humano, con uno u otro momento de la realidad. Tal vivencia es una vivencia de algo. No hay vivencias sin motivo, como no hay acto consciente que no fuera acto de conciencia de algo. Sin embargo, cada vivencia es personal (...). La verdadera unidad dinámica de la conciencia, unidad plena que constituye la base de la conciencia es la vivencia. (1984/1996, p. 383)

Según Vygotski, en una experiencia emocional (parezhivaniya) "siempre estamos frente a una unidad indivisible de las características personales y de las características situacionales las cuales se representan en la experiencia emocional (parezhivaniya) o vivencia” (Vygotski, 1935, p. 8). Hay que aclarar que en su concepción la vivencia incluye no solamente la emoción y los aspectos afectivos, sino que es la unión de estos con los cognitivos e intelectuales (Esteban, 2011). La vivencia determina entonces desde su punto de vista "de qué modo influye sobre el desarrollo del niño uno u otro aspecto del medio” (1984/1996, p. 383) y cómo se articulan ambos:

Vemos, pues, que en la vivencia se refleja, por una parte, el medio en su relación conmigo y el modo que lo vivo y, por otra se ponen de manifies- 
to las peculiaridades del desarrollo de mi propio “yo". En mi vivencia se manifiestan en qué medida participan todas mis propiedades que se han formado a lo largo de mi desarrollo en un momento determinado. (1984/1996b, p. 383)

Vygotski concluye afirmando que "si tuviéramos que formular una tesis formal, un tanto generalizada, será correcto decir que el medio determina el desarrollo del niño a través de la vivencia de dicho medio" (1984/1996, p. 383) y poniendo de relieve el papel activo de la persona en este proceso:

El niño es una parte de la situación social, su relación con el entorno y la relación con éste se realiza a través de la vivencia y de la actividad del propio niño; las fuerzas del medio adquieren significado orientador gracias a las vivencias del niño. (1984/1996, pp. 383-384)

Posteriormente en Pensamiento y lenguaje (1982/1993), vuelve sobre estas ideas, en esta ocasión para señalar en el inicio del trabajo donde aborda cuestiones metodológicas que "la primera cuestión que se plantea cuando hablamos de la relación entre el pensamiento y el lenguaje y los restantes aspectos de la conciencia es la de la conexión entre el intelecto y el afecto” (1982/1993, p. 24).

La relevancia de los aspectos afectivos y motivacionales para la comprensión de la construcción de significados como vínculo entre mente y cultura -para comprender en definitiva el paso de lo interpsicológico a lo intrapsicológico-, ha despertado también el interés entre los autores que trabajan en la perspectiva sociocultural, conscientes como Vygotski de la necesidad de integración de lo cognitivo y afectivo en la teoría y en la investigación. Esta inquietud se manifiesta desde hace un tiempo, por ejemplo, haciendo referencia al sentido en los planteamientos teóricos (Coll, 1988; Vila, 1995, 2013; Solé 1993) y más recientemente en el análisis de la práctica educativa en relación a la identidad (De la Mata y Santamaría, 2010; Coll y Falsafi, 2008, 2010; Falsafi y Coll, 2011; Kolb y Kolb, 2009; Ramírez, 2011; Rebollo y Hornilla, 2010; Rivas et al., 2010; Vila et al., 2010; Zarandona, 2015). De todas formas, tanto en tiempos de Vygotski como hoy en día, siguen siendo los aspectos menos estudiados, aunque hoy como entonces haya empezado a plantearse la necesidad de abordarlos.

\section{Problema, Objetivo y Preguntas de Investigación}

Lo que se presenta es una investigación en curso con la que se pretende contribuir al estudio de los contextos de psicoterapia como contextos de desarrollo abordando el siguiente problema de investigación: ¿de qué manera contribuye el estudio de la interacción social de las sesiones de psicoterapia organizada en base a los métodos relacionales de la Psicoterapia Integrativa en un contexto de formación experiencial de psicoterapeutas al estudio y la comprensión del desarrollo humano?

El objetivo de la investigación es hacer un análisis de la teoría y de la práctica de la Psicoterapia Integrativa en los seminarios observados que permita desvelar de qué manera contribuye al estudio y comprensión del desarrollo humano. Las 
preguntas de la investigación son las siguientes: ¿en qué confluyen, si es que lo hacen, la Psicoterapia Integrativa y la perspectiva constructivista sociocultural del desarrollo? esto es: ¿qué aspectos de la Psicología Evolutiva, y en concreto de la perspectiva constructivista y sociocultural de esta disciplina, se encuentran en los postulados teóricos y en la interacción social de la práctica clínica de la Psicoterapia Integrativa en el contexto de formación estudiado? ¿Qué aporta la Psicoterapia Integrativa a la investigación sobre el desarrollo humano desde dicha perspectiva?

\section{Metodología}

\section{Diseño de la Investigación}

Este trabajo se ha llevado a cabo desde un enfoque nombrado de distintas maneras -cualitativo/constructivista, etnográfico, estudio de casos-y al que aquí, siguiendo a Erickson (1989), se denominará interpretativo. Esta denominación incluye todo el conjunto de enfoques de la investigación observacional participativa. La aproximación empírica de esta investigación se centra, por tanto, en el análisis de documentos y en la observación, registro y análisis en distintos niveles de profundidad de, por una aparte, un número reducido de eventos interactivos de psicoterapia y, por otra, de todas las actividades desarrolladas en el seminario en las que se insertan éstos.

\section{Participantes}

Las personas de la investigación han sido los participantes en los seminarios experienciales de formación en Psicoterapia Integrativa (15 seminarios residenciales de 4 y 5 días de duración) y didácticos (4 didácticos también residenciales y de la misma duración). El número de participantes ha sido entre 20-25 personas incluidos los coterapeutas. Cabe destacar que estos últimos tienen una amplia y reconocida experiencia en la práctica de la Psicoterapia Integrativa. Han sido en todos los casos Richard Erskine (creador de esta línea de psicoterapia) y Amaia Mauriz (directora del Instituto BIOS de formación en Psicoterapia Integrativa y del máster en Psicoterapia Relacional Integrativa de la Universidad de Deusto).

En los seminarios experienciales se trabaja fundamentalmente a partir de las sesiones de terapia individual dentro del grupo que plantean los participantes, seguidas de discusión teórica y metodológica. En los seminarios didácticos priman las explicaciones teóricas si bien se intercala alguna sesión de terapia como ejemplificación de lo explicado.

\section{Herramientas de Selección y Recogida de Datos}

Éstas han sido la observación participante, análisis de documentos (libros y artículos sobre conceptos y métodos de la Psicoterapia Integrativa ejemplificados con transcripciones de casos) y el registro literal de sesiones de terapia en las que se han recogido, además de lo que dicen y hacen los participantes, notas sobre 
los elementos no verbales de la comunicación (risa, llanto, gestos, movimientos, postura corporal, tono y velocidad de la voz, uso del espacio). El hecho de trabajar con traducción sucesiva ha permitido poder anotar fielmente todas las emisiones de los participantes cuando no se han tomado registros de audio.

\section{Tratamiento y Análisis de Datos}

Las notas recogidas se han organizado por fecha y temática de las mismas, en un texto digital; transcripción de los registros de audio de las sesiones y elaboración de un texto digital con todas las transcripciones. En cuanto al análisis: análisis de contenido de los documentos en base a las preguntas de la investigación y análisis de las pautas de actuación de los participantes en la interacción terapéutica a partir de las categorías conceptuales de la perspectiva constructivista sociocultural.

\section{Resultados}

Lo que se presenta es el resultado de una primera aproximación al tratamiento y análisis de los datos. En cuanto a los aspectos de la perspectiva constructivista y sociocultural presentes en la teoría y la práctica de la Psicoterapia Integrativa se ha encontrado lo siguiente:

\section{La Conceptualización del Desarrollo}

Tanto en los documentos como en la práctica clínica se observa que el proceso evolutivo se considera, al igual que en la perspectiva constructivista sociocultural explicada más arriba, fruto tanto de la acción del sujeto, como de las interacciones y de los contextos de desarrollo en los que ha participado y/o participa. Esta comprensión se aprecia especialmente en la visión no patologizante de los problemas que presenta la persona que acude a psicoterapia: para la Psicoterapia Integrativa, la carencia o patología se encuentra en los contextos y formas de interacción en los que las personas han participado, las cuales han tenido que desarrollar como solución de supervivencia un determinado funcionamiento psicológico que puede ser disfuncional y fuente de sufrimiento en la actualidad, una visión que aparece repetidamente en las explicaciones teóricas y análisis de casos de los seminarios. Esta visión puede apreciarse igualmente en las explicaciones de los conceptos básicos de la Psicoterapia Integrativa en diferentes documentos, por ejemplo, en Erskine et al. (1999/2012, p. 36) al hablar sobre "La relación y el hacerse humanos” y que se traduce expresamente en los métodos: en el uso de la validación, -“reconocimiento de la importancia de la experiencia del cliente” (Erskine et al., 1999/2012, p. 145) y de la normalización, -“el propósito de la normalización [...] es ayudar a los clientes a darse cuenta de que sus comportamientos y sus experiencias internas son respuestas normales y predecibles a su situación vital, a su entorno y a su composición genética” (p. 149). En los registros de las sesiones de terapia en los seminarios aparecen a menudo en las intervenciones del psicoterapeuta en la interacción en relación al relato de la experiencia de la persona en contextos de 
desarrollo violentos o negligentes, expresiones encaminadas a la validación y normalización tales como “¿y qué hiciste para no volverte loco?”, formulación que en sí misma subraya el valor de supervivencia de la experiencia interna de la persona.

La concepción del desarrollo de la que hablamos se aprecia también en el punto de vista evolutivo como guía y referencia de la práctica clínica que propugna esta línea de psicoterapia. Se trabaja en todo momento teniendo en cuenta el conocimiento sobre el desarrollo humano en general: "Los conceptos se emplean desde una perspectiva de evolución humana, en la que cada fase de la vida presenta tareas de desarrollo específicas, sensibilidad hacia ciertas necesidades, crisis y oportunidades de realizar nuevos aprendizajes” (Erskine y Moursund, 1988/2014, pp. 53-54). Y se trabaja teniendo en cuenta también el proceso evolutivo particular de la persona y la fase del mismo en que se encuentra en cada momento del proceso terapéutico: "Es natural que en esta etapa del desarrollo persista un sentido de omnipotencia, aunque difuso. El Chris de cinco años cree..." [en referencia a una intervención psicoterapéutica con un adulto] (Erskine y Moursund, 1988/2014, p. 100). Por otro lado, el énfasis del “contacto en relación” y de los métodos relacionales base del trabajo terapéutico, se apoya en la literatura sobre el desarrollo en la que aparece la importancia de los vínculos para el mismo (Erskine, 2015).

\section{Estudio y Cuidado de la Interacción}

Coherente con esta concepción del desarrollo y con los métodos relacionales fundamentados en la misma, se ha podido observar tanto en los seminarios como en los textos publicados para explicar los métodos, una especial atención a las actuaciones de los participantes, -psicoterapeuta y cliente-, en la interacción que se establece entre ambos en las sesiones de terapia. Se potencian los registros de las sesiones tanto en los seminarios de formación como en las sesiones fuera de ese contexto para su posterior análisis, análisis en la que se estudia las pautas de actuación de los protagonistas y su articulación en la interacción (en las discusiones de los seminarios, en los textos publicados), y las actuaciones del terapeuta que sirven de ayuda al cliente (Erskine, 1996, 1997, 2015) al modo de las investigaciones realizadas desde la perspectiva sociocultural del desarrollo sobre el funcionamiento interpsicológico y sobre el paso del funcionamiento interpsicológico al intrapsicológico que hemos visto. Se estudia por ello especialmente la cualidad de la interacción que sirve al proceso terapéutico, especificándose muy concretamente, y elaborando categorías para ello, las características de las actuaciones del terapeuta extraídas de la investigación sobre la práctica clínica, (análisis de casos), a tener en cuenta y ejemplificadas siempre con transcripciones de la interacción de las sesiones de terapia. Así se considera que indagación, sintonización e implicación son los "tres aspectos del comportamiento del psicoterapeuta que componen la esencia de una relación terapéutica efectiva” (Erskine et al., 1999/2012, p. 51) y se precisan características de la indagación terapéutica y técnicas para llevarla a cabo, tipos de sintonización y formas de transmitirla así como características de la 
implicación y formas de expresarla (Erskine y Moursund, 1988/2014; Erskine et al., 1999/2012). Toda esta profundización en el conocimiento de las actuaciones de los terapeutas en la interacción relevantes para el proceso terapéutico se entiende si tenemos en cuenta que:

Los psicoterapeutas integrativos enfocados a la relación consideran que dicha relación terapéutica ejerce una influencia curativa de varias maneras. Una de ellas tiene que ver con la precisión, la exactitud de la respuesta empática del/la terapeuta y la agudeza de su sintonía con su cliente. (Moursund y Erskine, 2004, p. 11)

Por otra parte, la asunción, de que tal como se sostiene desde los postulados vygotskianos, la estructura del funcionamiento interpsicológico tiene un enorme impacto sobre la estructura del funcionamiento intrapsicológico resultante, se plasma igualmente en afirmaciones de la Psicoterapia Integrativa tales como: "El tipo de relación que los terapeutas crean, y la habilidad con la cual la utilicen, será un determinante primario en el éxito o en el fracaso de la empresa terapéutica” (Moursund y Erskine, 2004, p. 9) y “(...) en el contexto de esta relación podemos aportar un modelo acerca de la clase de proceso en el cual nos gustaría que el cliente se implicase” (p. 11).

En los registros de las sesiones de los seminarios las personas en más de una ocasión expresan el impacto que ha tenido en ellas las actuaciones del terapeuta en su relación.

Finalmente, al igual que en la perspectiva sociocultural, se tiene muy presente la importancia de la acción del propio sujeto: todas las actuaciones del psicoterapeuta en la interacción van encaminadas a potenciar y respetar su iniciativa y a estimular su participación en el proceso.

\section{Ajuste de la Ayuda}

La importancia de que la ayuda terapéutica para ser efectiva parta siempre del nivel en que se encuentra la persona en ese momento de su desarrollo se refleja en la importancia que se concede en los métodos de la Psicoterapia Integrativa a la sintonización, insistiendo en que ha de ser tanto cognitiva como afectiva y que ésta última supone sintonización rítmica y sintonización con la etapa evolutiva (Erskine et al., 1999/2012), algo que como hemos visto más arriba, es necesario para que la persona avance en la zona de desarrollo próximo: “Tan sorprendente experiencia [de relación] le permite ir más allá” (Moursund y Erskine, 2004, p. 12). Puede decirse que el terapeuta actúa como el adulto en los procesos de participación guiada en los cuales como nos decía Rogoff (1990/1993, p. 142):

Éste ajusta el grado de apoyo durante la interacción en base a las "pistas" que aparecen en la misma de forma que le hace posible alcanzar "una estructura desafiante y, a la vez, de apoyo, que se ajuste a los cambios en la comprensión del aprendiz. (p. 142)

De forma parecida se expresan en la Psicoterapia Integrativa: 
Con el soporte del/la terapeuta puede re-examinar sus formas de ser y estar con otras personas, y de pensar acerca de otros y acerca de sí. Con el respeto y cuidado genuino del terapeuta puede empezar a cambiar algunas de estas formas de “estar con” y de pensar sobre. (Moursund y Erskine, 2004, p. 13)

\section{Especial Atención a la Vivencia}

En los seminarios tanto en las sesiones de terapia como en las discusiones teóricas y metodológicas aparece la importancia de considerar sobre todo la experiencia fenomenológica de la persona. Se subraya, para la propia toma de conciencia de la persona y para la formación de los psicoterapeutas, que más determinante para el desarrollo que lo que sucedió, es cómo lo vivió el sujeto. Un planteamiento en la línea de lo que hemos visto sobre la vivencia en el apartado sobre la perspectiva sociocultural. Acorde con él se insiste y se dan pautas precisas para una indagación fenomenológica respetuosa.

En cuanto a las aportaciones de la Psicoterapia Integrativa a la investigación del desarrollo desde la perspectiva constructivista sociocultural:

\section{Investigación sobre Cualidad Afectivo Emocional de la Interacción}

En los estudios sobre la interacción social desde la perspectiva sociocultural, se ha prestado poca atención a la cualidad afectivo emocional de la interacción y su papel en el paso del funcionamiento interpsicológico al intrapsicológico. Los trabajos de la Psicoterapia Integrativa acerca de las necesidades relacionales y las actuaciones de los psicoterapeutas en la interacción que sirven de ayuda al proceso terapéutico mencionadas más arriba son una aportación para empezar a cubrir esa necesidad que se plantea en la perspectiva sociocultural de integración de lo cognitivo y afectivo en la teoría y en la investigación.

\section{Investigación sobre la Acción y la Vivencia del Sujeto}

Si bien como hemos visto, la perspectiva constructivista sociocultural postula la importancia de la acción del sujeto en el paso del funcionamiento interpsicológico al intrapsicológico (proceso de internalización o apropiación) así como la de su vivencia, lo cierto es que la investigación hasta ahora ha estado orientada al estudio del funcionamiento interpsicológico y existen muchas dificultades metodológicas para poder acceder a la acción externa e interna de la persona en el proceso de internalización y a su vivencia. La Psicoterapia Integrativa a través de la indagación histórica y fenomenológica respetuosa y realizada en sintonía y con implicación permite este acceso, no solamente a través del relato de la persona, sino también a la observación de las actuaciones de la misma en la interacción, -en la relación terapéutica- en la que reproduce de forma automática la acción que realizó, así como la forma de vivirla, todo lo cual puede observarse tanto en los registros de las sesiones de terapia que aparecen en las diferentes publicaciones sobre Psicoterapia Integrativa como en las sesiones registradas en los seminarios. 
Un rico material sobre el que merece la pena seguir investigando.

\section{Conclusiones}

Como conclusiones puede decirse que hay varios aspectos de la perspectiva constructivista sociocultural del desarrollo que están presentes en los postulados teóricos y la práctica clínica de la Psicoterapia Integrativa, a saber, la propia comprensión del desarrollo de las personas; la atención y el cuidado a la interacción en la práctica clínica y en la investigación sobre la misma a partir de registros y análisis de las pautas de actuación de los participantes; el tener en cuenta el ritmo y el desarrollo efectivo de las personas a la hora de intervenir como requisito ineludible para propiciar el avance de las personas en el proceso terapéutico y en su desarrollo (zona de desarrollo próximo); el ajuste de la ayuda que proporciona el psicoterapeuta durante la interacción a lo que necesita la persona; el cuidado de la cualidad de la interacción y, por último, la importancia que se da a la acción del sujeto y a su vivencia.

Por otro lado, puede decirse que los métodos relacionales de la Psicoterapia Integrativa son una aportación a la investigación y comprensión del desarrollo humano en el campo del estudio de la cualidad afectivo emocional de la interacción y su relación con el paso del funcionamiento interpsicológico al intrapsicológico; una aportación igualmente a la investigación sobre la acción del sujeto en dicho paso (proceso de internalización o apropiación) y finalmente, una aportación al estudio de la vivencia del sujeto en dichos procesos.

\section{Referencias}

Arias, G. (2015). Contribuciones de los cubanos a lo Histórico-Cultural. Un debate constituyente. Alternativas cubanas en psicología, 3(7), 13-24. https:/www.acupsi.org/articulo/84/contribuciones-de-los-cubanos-alo-histrico-cultural-un-debate-constituyente.html

Brofenbrenner, U. (1985). Contextos de crianza del niño. Problemas y perspectivas (A. Alvarez, Trad.). Infancia y Aprendizaje, 29, 45-55. (Trabajo original publicado en 1979)

Brofenbrenner, U. (1987). La ecología del desarrollo humano (A. Devoto, Trad.). Paidós. (Trabajo original publicado en 1979)

Bruner, J. (1986). El habla del niño (R. Premat, Trad.). Paidós. (Trabajo original publicado en 1983)

Bruner, J. (1991). Actos de significado (J. C. Gómez Crespo y J. Linaza, Trad.). Alianza. (Trabajo original publicado en 1990)

Bruner, J. (1997). La educación puerta de la cultura (F. Díaz, Trad.). Visor.

Bruner, J. (2008). Culture and mind: Their fruitful incommensurability [Cultura y mente: Su fructífera incomensurabilidad]. Ethos, 36, 29-45. https://doi.org/10.1111/j.1548-1352.2008.00002.x

Coll, C. (1988). Significado y sentido en el aprendizaje escolar. Reflexiones en torno al concepto de aprendizaje significativo. Infancia y Aprendizaje, 11(41), 131-142. https://doi.org/10.1080/02103702.1988.10822196

Coll, C. y Solé, I. (1990). La interacción profesor/alumno en el proceso de enseñanza y aprendizaje. En C. Coll, J. Palacios y A. Marchesi (Comps.), Desarrollo psicológico y educación. Vol. II. Psicología de la educación (pp. 315-333). Alianza.

Coll, C. y Falsafi, L. (2010). Learner identity. An educational and analytical tool [Identidad del alumno. Una herramienta educativa y analítica]. Revista de Educación, 353, 211-233. http://www.revistaeducacion. educacion.es/re353/re353_08.pdf

Cubero, R. (2005). Perspectivas constructivistas. Graó. 
De la Mata, M. L. y Santamaría, A. (2010). La construcción del yo en escenarios educativos. Un análisis desde la psicología cultural. Revista de Educación, 353, 157-186. http://www.revistaeducacion.educacion.es/ re353/re353_06.pdf

Erickson, F. (1989). Métodos cualitativos de investigación sobre la enseñanza. En M. Wittrok (Ed.), La investigación de la enseñanza II. Métodos cualitativos de observación. (pp. 203-247). Paidós MEC.

Erskine, R. G. (1996). Necesidades relacionales. Instituto de Psicoterapia Integrativa. https://www.integrativetherapy.com/es/articles.php?id=21

Erskine, R. G. (1997). Methods of an Integrative Psychotherapy [Métodos de una psicoterapia integrativa]. En R. G. Erskine (Ed.), Theories and Methods of an Integrative Transactional Analysis. A Volume of Selected Articles (pp. 28-29). TA.

Erskine, R. G. (2015). Attachment, Relational-needs and the child's processes of atribution of meaning and sense making [Apego, necesidades relacionales y los procesos de atribución de significado y búsqueda de significado en niños] [Manuscrito no publicado].

Erskine, R. G., Moursund, J. y Trautmann, R. (2012). Más allá de la empatía (N. Mestre, Trad.). Descleé de Brouwer. (Trabajo original publicado en 1999)

Erskine, R. G. y Moursund, J. P. (2014). La Psicoterapia Integrativa en Acción (I. Arregui, Trad.). Desclée de Brouwer. (Trabajo original publicado en 1988)

Esteban, M. (2011). Una interpretación de la Psicología Cultural: aplicaciones prácticas y principios teóricos. Suma Psicológica, 18(2), 65-88.

Falsafi, L. y Coll, C. (2011). La construcción de identidad de aprendiz. Coordenadas espacio-temporales. En C. Monereo y J. I. Pozo (Ed.), La identidad en Psicología de la Educación (pp.77-99). Narcea.

Fariñas, G. (1999a). Acerca del concepto de Vivencia en el enfoque Histórico-cultural. Revista Cubana de Psicología, 16(3), 171-176. http://pepsic.bvsalud.org/pdf/rcp/v16n3/11.pdf

Fariñas, G. (1999b). L. S. Vigotsky en la cultura y la subjetividad del psicólogo. Revista Cubana de Psicología, 16(3), 235-240. http://pepsic.bvsalud.org/pdf/rcp/v16n3/13.pdf

Fariñas, G. (2013). Lo emblemático en el enfoque Histórico Cultural: apuntes para una praxis de rigor. Amazónica, 11(2), 102-134.

Fariñas, G. (2015). Acerca del pensamiento histórico culturalista desde la perspectiva de los psicólogos y pedagogos cubanos. Alternativas cubanas en Psicología, 7(3), 25-36. http://www.alfepsi.org/alternativas-cubanas-enpsicologia-vol-3-num-7-2015/

Kaye, K. (1986). La vida mental y social del bebé (D. Roesenbaum, Trad.). Paidós. (Trabajo original publicado en 1982)

Kolb, A. y Kolb, D. (2009). On Becoming a Learner: The Concept of Learning Identity [Conviertiéndose en un alumno. El concepto de la identidad del aprendizaje]. En D Bamford-Rees, B. Doyle, B. Klein-Collins y J. Wertheim (Eds.), Learning never ends: Essays on adult learning inspired by the life and work of David O. Justice (pp. 5-13). CAEL Forum and News.

Leontiev, A. (1983). El desarrollo del psiquismo (3a ed., E. Calatayud, Trad.). Akal.

Marcos, B. (2005). Estudio del desarrollo de la identidad profesional del maestro. Un enfoque teórico y una propuesta de intervención (Tesis doctoral, Universidad de La Habana). http://www.biblioteca.uh.cu/ servicios/deposito-tesis

Martí, E. (2005). Desarrollo, cultura y educación. Amorrortu editores.

Mercer, N. (2001). Palabras y mentes (G. Sánchez Barberán, Trad.). Paidós. (Trabajo original publicado en 2000)

Moursund, J. P. y Erskine, R. G. (2004). Integrative Psychoterapy. Art and science of relationship [Psycoterapia integrative, arte y ciencia de las relación]. T Books.

Ramírez, D. (2011). La identidad en tiempos de cambio. Una aproximación sociocultural. En C. Monereo y J. I. Pozo (Eds.), La identidad en Psicología de la Educación (pp. 27-45). Narcea.

Rebollo, M. A. y Hornilla Gómez, I. (2010). Perspectiva emocional en la construcción de la identidad en contextos educativos: discursos y conflictos emocionales. Revista de Educación 353, 235-263. http://www.revistaeducacion.educacion.es/re353/re353_09.pdf

Rivas, J. I., Leite, A. E., Cortés, P, Márquez, M. J. y Papua, D. (2010). La configuración de identidades en la experiencia escolar. Escenarios, sujetos y regulaciones. Revista de Educación, 353, 187-209. http://www.revistaeducacion.educacion.es/re353/re353_07.pdf

Rogoff, B. (1993). Aprendices del pensamiento (P. Lacasa, Trad.). Paidós. (Trabajo original publicado en 1990)

Rogoff, B. (1997). Los tres planos de la actividad sociocultural: apropiación participativa, participación guiada y aprendizaje (F. Blanco Trejo y M. J. de Tiena Dávila, Trad.). En J. V. Wertsch, P. del Río y A. Álvarez (Eds.), La mente sociocultural (pp. 111-128). Fundación Infancia y Aprendizaje. 
Rogoff, B. (2012). Learning without lessons: Opportunities to expand knowledge [Aprendizaje sin lecciones: Oportunidades para ampliar el conocimineto]. Infancia y Aprendizaje, 35(2), 233-252. https://doi. org/10.1174/021037012800217970

Santamaría, A. (2004). ¿Es posible el diálogo entre la mente y la cultura? Hacia una psicología cultural de la mente. Suma Psicológica, 11(2), 247-266.

Solé, I. (1993). Disponibilidad del aprendizaje y sentido del aprendizaje. En C. Coll, E. Martín, T. Mauri, M. Miras, J. Onrubia, I. Solé y A. Zabala (Eds.), El constructivismo en el aula (pp. 25-47). Graó.

Vila, I. (1995). Psicología de la Educación. Proyecto docente para Concurso-Oposición de Cátedra de Psicología Evolutiva y de la Educación [Manuscrito no publicado]. Departament de Psicologia, Facultat de Psicologia, Universitat de Girona.

Vila, I. (1996). La idea de los orígenes sociales de las funciones psicológicas no es antitética con la noción de construcción personal. Anuario de Psicología, 69, 82-92. https://www.raco.cat/index.php/AnuarioPsicologia/article/view/61314/88948

Vila, I. (2013). Presentación proyecto de investigación sentido e identidad de aprendiz en el proceso de escolarización del alumnado extranjero en Cataluña [Manuscrito no publicado]. Departamento de Psicología, Facultad de Psicología, Universidad de Girona.

Vila, I., Esteban, M. y Oller, J. (2010). Identidad nacional, lengua y escuela. Revista de Educación, 353, 39-65. http://www.revistaeducacion.educacion.es/re353/re353_02.pdf

Vygotski, L. S. (1935). El problema del entorno [Manuscrito no publicado]. Catedra de Vygotski, Facultad de Psicología de La Habana, Ciudad de la Habana, Cuba.

Vygotski, L. S. (1979). El desarrollo de los procesos psicológicos superiores (S. Firió, Trad.). Grijalbo. (Trabajo original publicado en 1978)

Vygotski, L. S. (1991a). El método instrumental en psicología (J. M. Bravo, Trad.). Obras Escogidas Vol I (pp.6571). Visor y MEC. (Trabajo original publicado en 1982)

Vygotski, L. S. (1991b). El significado histórico de la crisis de la psicología (J. M. Bravo, Trad.). Obras Escogidas Vol I (pp.257-413). Visor y MEC. (Trabajo original publicado en 1982)

Vygotski, L. S. (1993). Pensamiento y lenguaje (J. M. Bravo, Trad.). Obras escogidas, Vol II (9-287). Visor y MEC. (Trabajo original publicado en 1982)

Vygotski, L. S. (1995). Historia del desarrollo de las funciones psíquicas superiores (L. Kuper, Trad.). Obras Escogidas Vol III (pp.11-341). Visor y MEC. (Trabajo original publicado en 1983)

Vygotski, L. S. (1996). La crisis de los siete años superiores (L. Kuper Trad.). Obras Escogidas Vol IV (pp. 377 386). Visor y MEC. (Trabajo original publicado en 1984)

Wertsch, J. (1988). La formación social de la mente (J. Zanón y M. Cortés, Trad.). Paidós. (Trabajo original publicado en 1985)

Wertsch, J. (1989). Semiotic mechanisms in joint cognitive activity [Mecanísmo semióticos en la actividad cognitiva conjunta]. Infancia y Aprendizaje, 47, 3-36. https://doi.org/10.1080/02103702.1989.10822239

Wertsch, J. (1993). Voces de la mente (A. Silvestre, Trad.). Aprendizaje Visor. (Trabajo original publicado en 1991)

Wertsch, J. (1997). La necesidad de la acción en la investigación sociocultural (F. Blanco Trejo y M. J. de Tiena Dávila, Trad.). En J. Wertsch, P. Del Río y A. Alvarez, (Eds.), La mente sociocultural (pp. 50-62). Fundación Infancia y Aprendizaje.

Wertsch, J. (1999). La mente en acción. Aique. (Trabajo original publicado en 1998)

Wood, D., Bruner, J. S. y Ross, G. (1976). The role of tutoring in problem solving [El rol del tutor resolviendo un problema]. Journal of Child Psychology and Psychiatry, 17(2), 89-100. https://doi.org/10.1111/j.1469-7610.1976. tb00381.x

Zarandona, E. (2007). De la espontaneidad a la repetición: análisis empírico de la interacción social en un aula de Educación Infantil. VI Congreso Iberoamericano de Psicodrama y conflictos culturales. Editorial Congrega.

Zarandona, E. (2011). ¿Necesita límites la espontaneidad? Boletín de la Sociedad Española de Psicoterapia y Técnicas de Grupo, 29, 35-63.

Zarandona, E. (2015). La construcción del escenario en la interacción social como transformación especifica de la actividad en Educación Infantil [Tesis doctoral, Universidad del País Vasco]. http://hdl.handle. net/10810/16414 